\title{
Adsorption separation of $\mathrm{Cr}(\mathrm{VI})$ from water phase by using multiwalled-carbon-nanotube-immobilized ionic liquids
}

Lihan Sun ${ }^{\dagger}$, Mengru Wang ${ }^{\dagger}$, Wei Li, Sha Luo, Yan Wu, Chunhui Ma*, Shouxin Liu**

Key Laboratory of Bio-based Material Science and Technology (Ministry of Education),

College of Material Science and Engineering, Northeast Forestry University, 150040 Harbin,

China

${ }^{\dagger}$ Authors contributed equally to this work.

Corresponding authors' address: Key Laboratory of Bio-based Material Science and

Technology (Ministry of Education), College of Material Science and Engineering, Northeast

Forestry University, Harbin 150040, China

*Tel.: +86-451-82191204, Fax: +86-451-82191204 (C. Ma)

**Tel.: +86-451-82191204, Fax: +86-451-82191502 (S. Liu)

*E-mail: mchmchmchmch@163.com (C. Ma) 
**E-mail: liushouxin@126.com (S. Liu) 

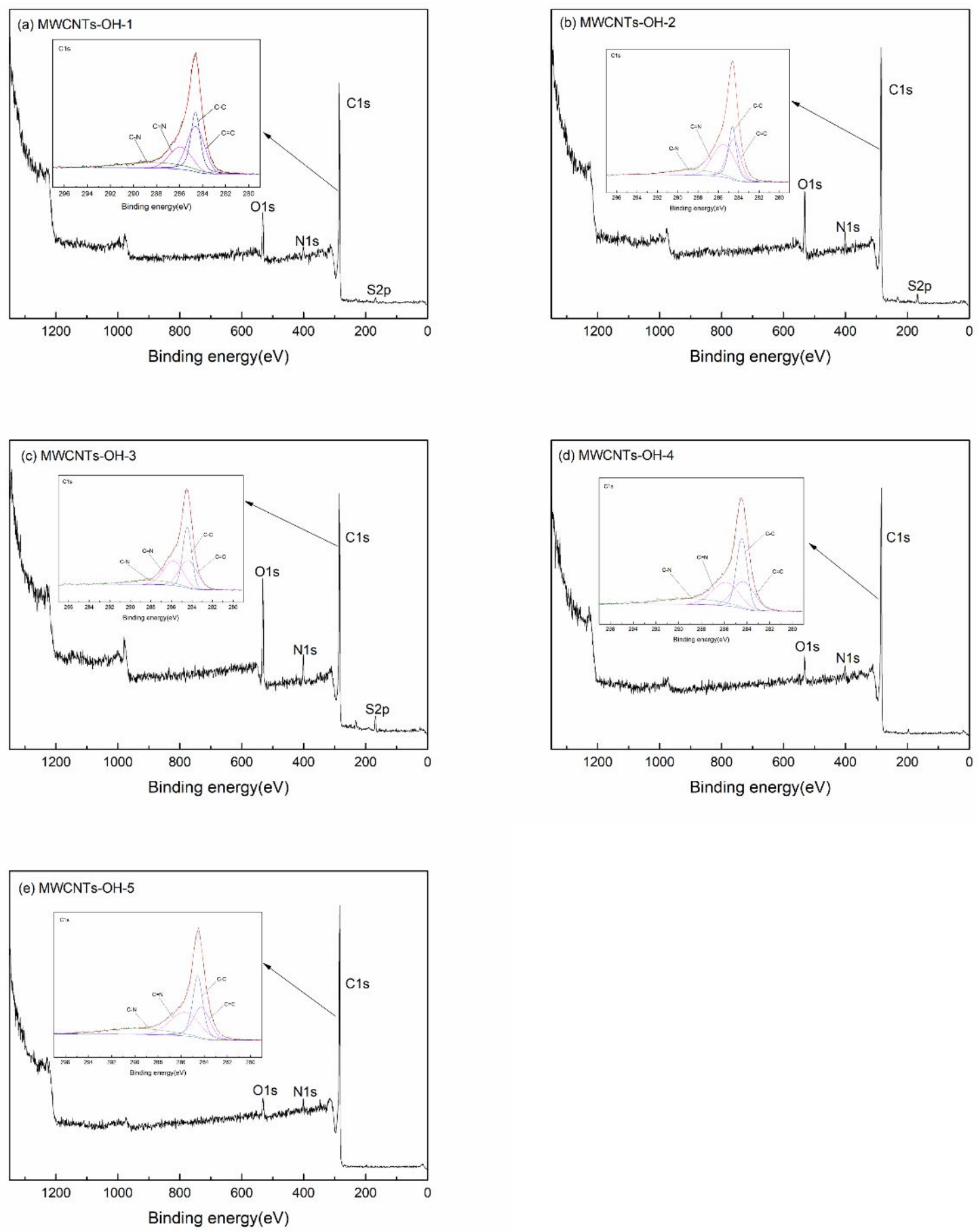

Figure S1 XPS spectra of MWCNTs-OH-1 immobilized ILs and C1s peak split spectra (a),

MWCNTs-OH-2 immobilized ILs and C1s peak split spectra (b), MWCNTs-OH-3 immobilized

ILs and C1s peak split spectra (c), MWCNTs-OH-4 immobilized ILs and C1s peak split spectra (d), MWCNTs-OH-5 immobilized ILs and C1s peak split spectra (e) 

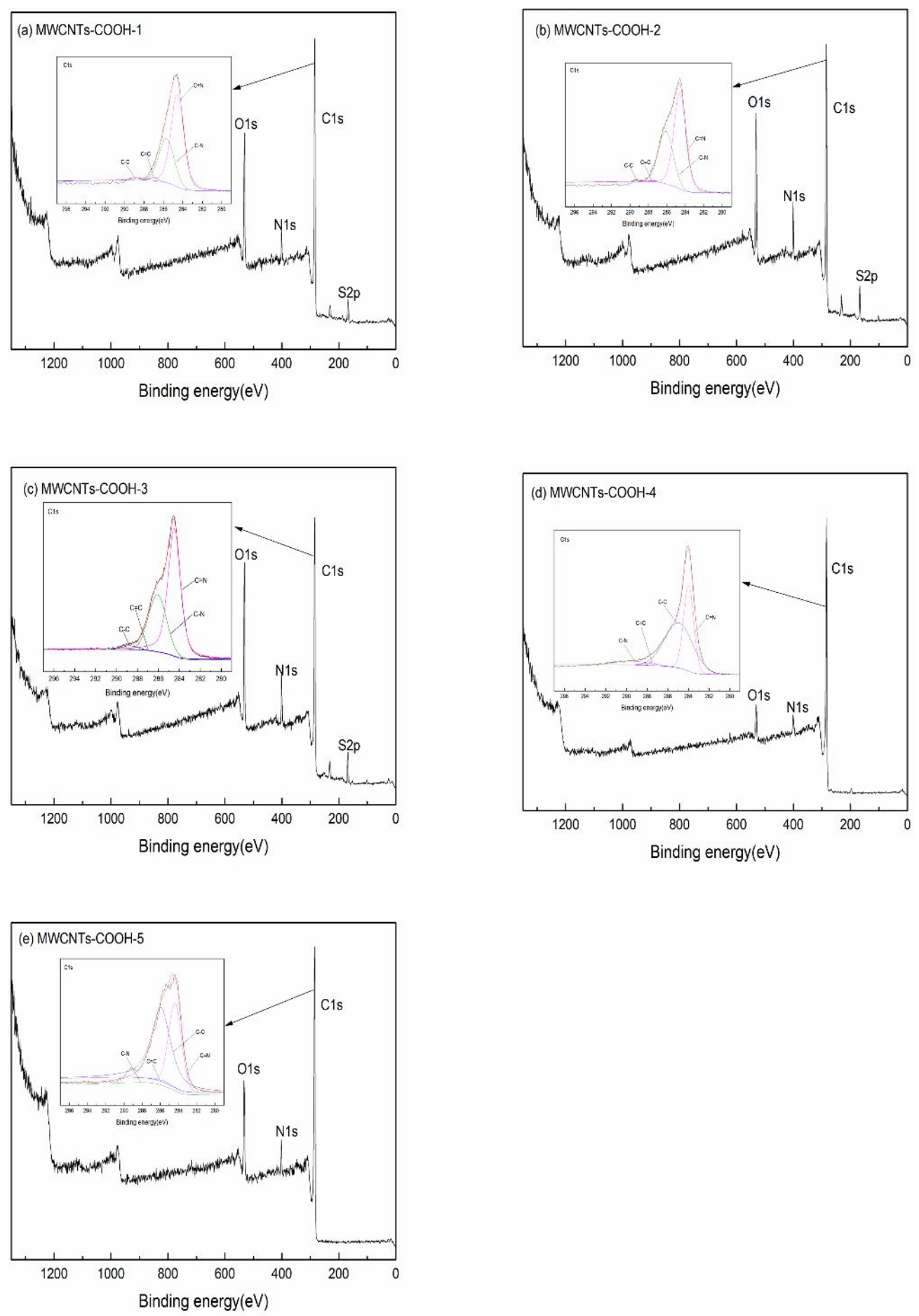

Figure S2 XPS spectra of MWCNTs-COOH-1 immobilized ILs and C1s peak split spectra (a),

MWCNTs-COOH-2 immobilized ILs and C1s peak split spectra (b), MWCNTs-COOH-3 
immobilized ILs and C1s peak split spectra (c), MWCNTs-COOH-4 immobilized ILs and C1s peak split spectra (d), MWCNTs-COOH-5 immobilized ILs and C1s peak split spectra (e)
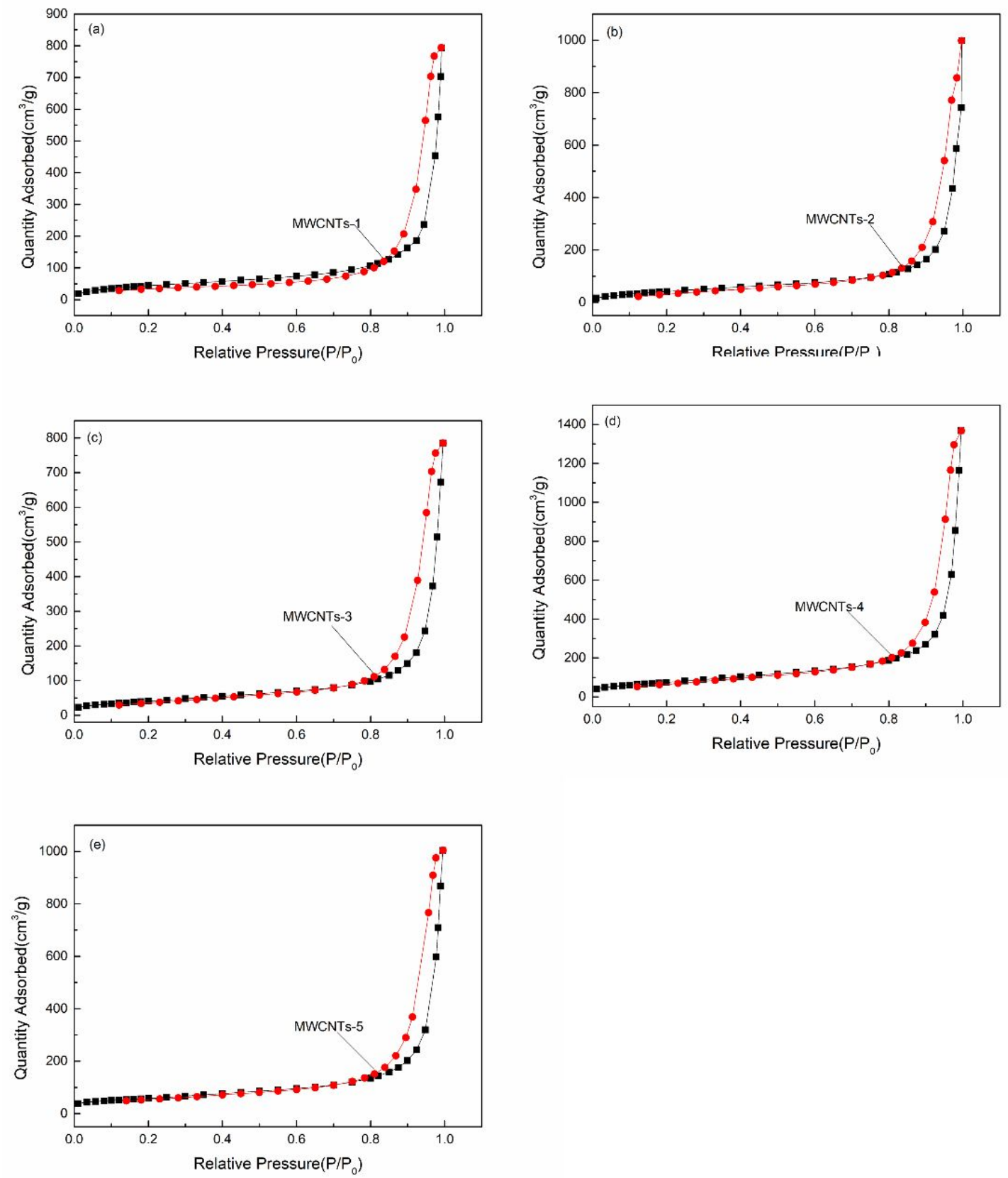
Figure S3 The $\mathrm{N}_{2}$ adsorption-desorption isotherms curves of MWCNTs-1 (a), MWCNTs-2

(b), MWCNTs-3 (c), MWCNTs-4 (d), MWCNTs-5 (e) 

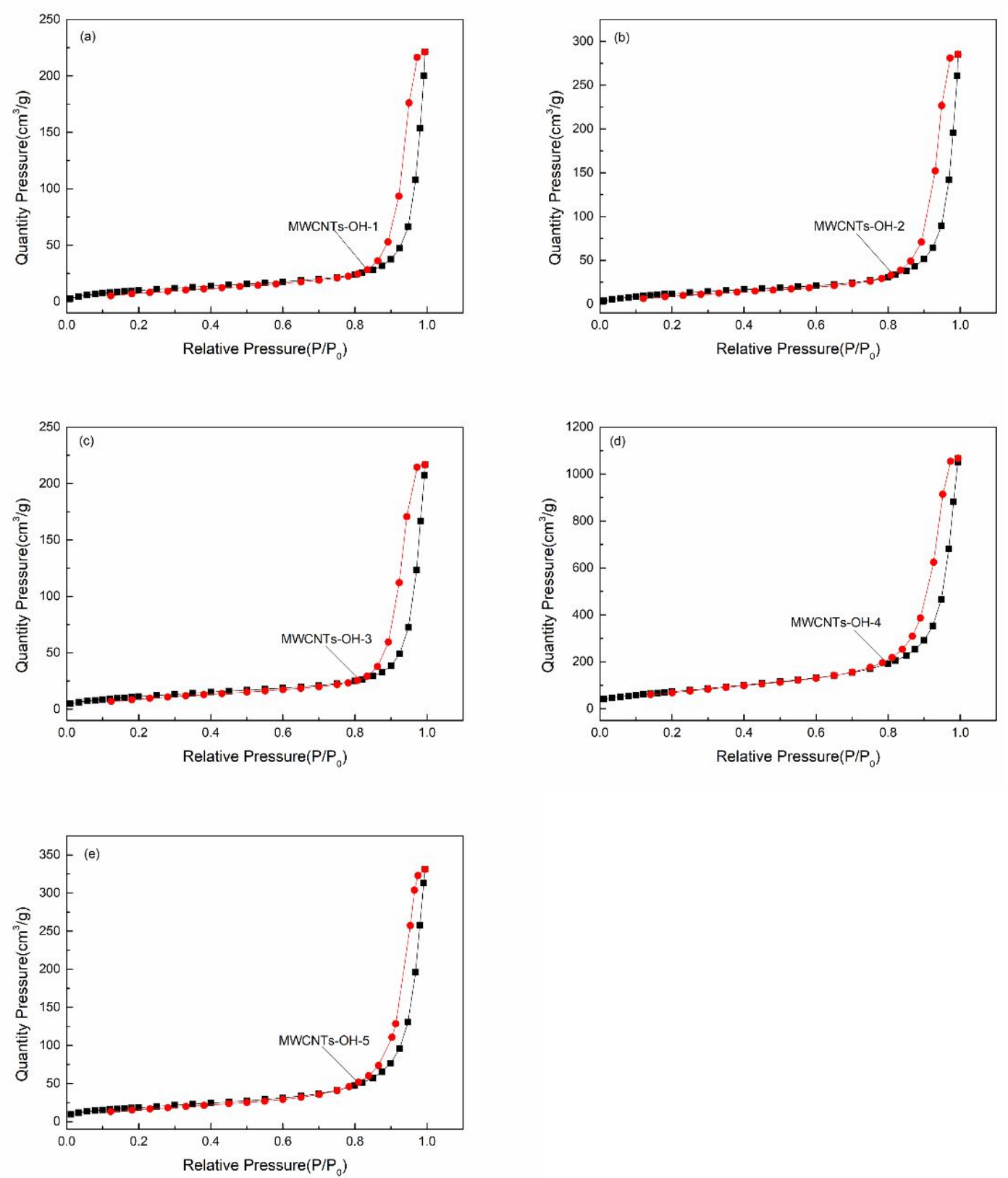

Figure S4 The $\mathrm{N}_{2}$ adsorption-desorption isotherms curves of MWCNTs-OH-1 (a), MWCNTsOH-2 (b), MWCNTs-OH-3 (c), MWCNTs-OH-4 (d), MWCNTs-OH-5 (e) 

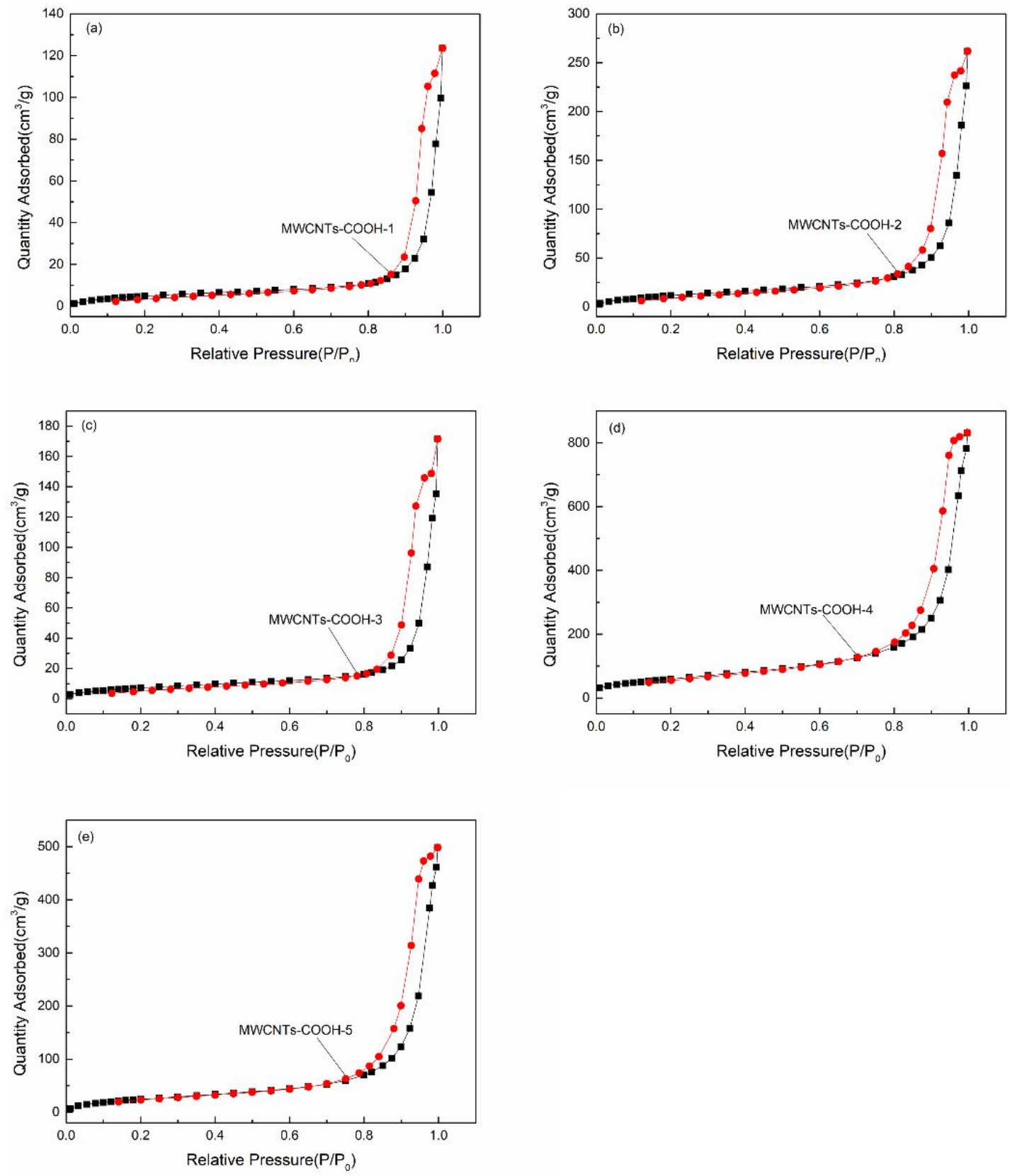

Figure S5 The $\mathrm{N}_{2}$ adsorption-desorption isotherms curves of MWCNTs-COOH-1 (a), 
MWCNTs-COOH-2 (b), MWCNTs-COOH-3 (c), MWCNTs-COOH-4 (d), MWCNTs-COOH-5

(e) 

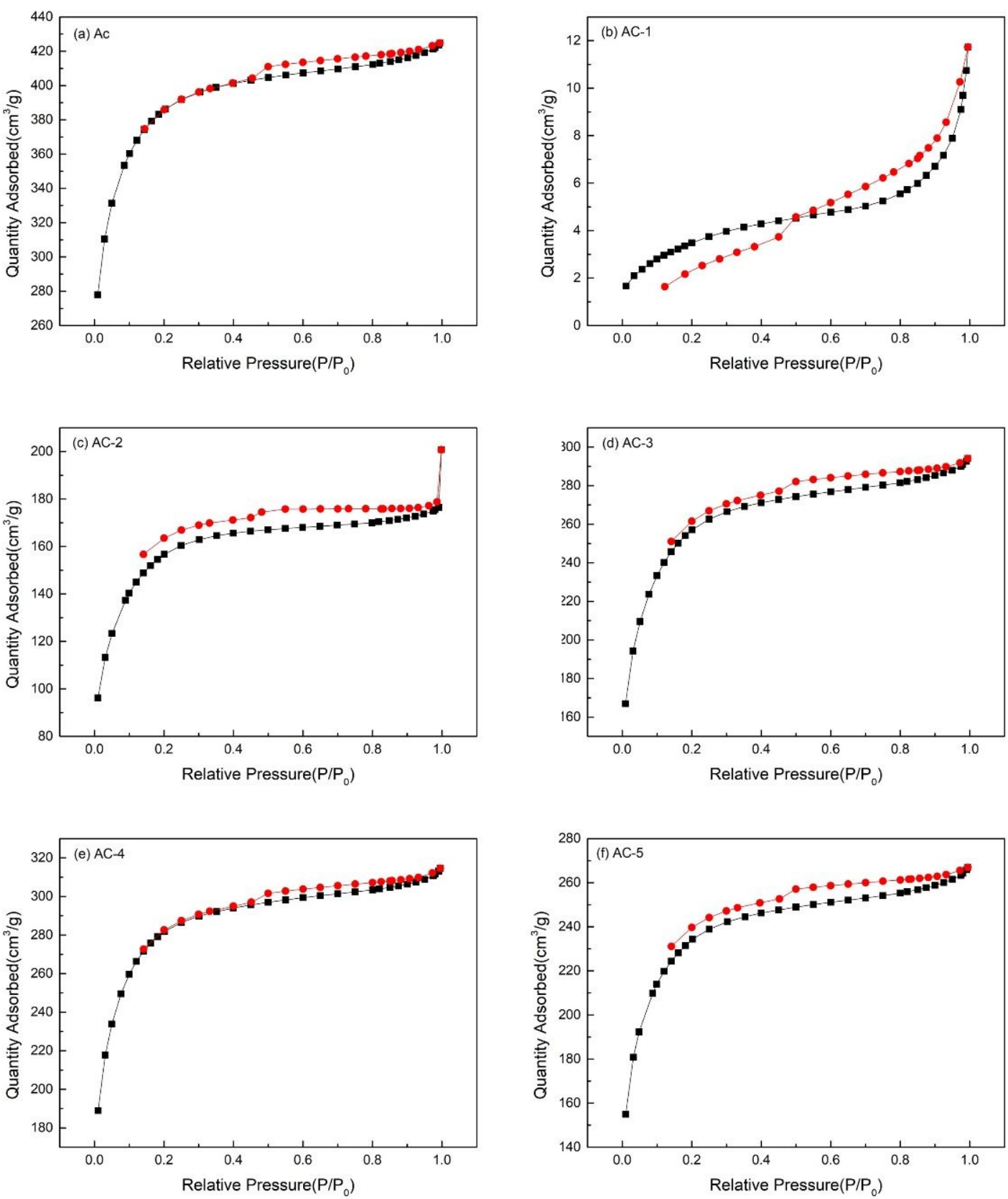

Figure $\mathbf{S 6}$ The $\mathrm{N}_{2}$ adsorption-desorption isotherms curves of AC (a), AC-1 (b), AC-2 (c), AC-

$3(\mathrm{~d}), \mathrm{AC}-4(\mathrm{e}), \mathrm{AC}-5$ (f) 

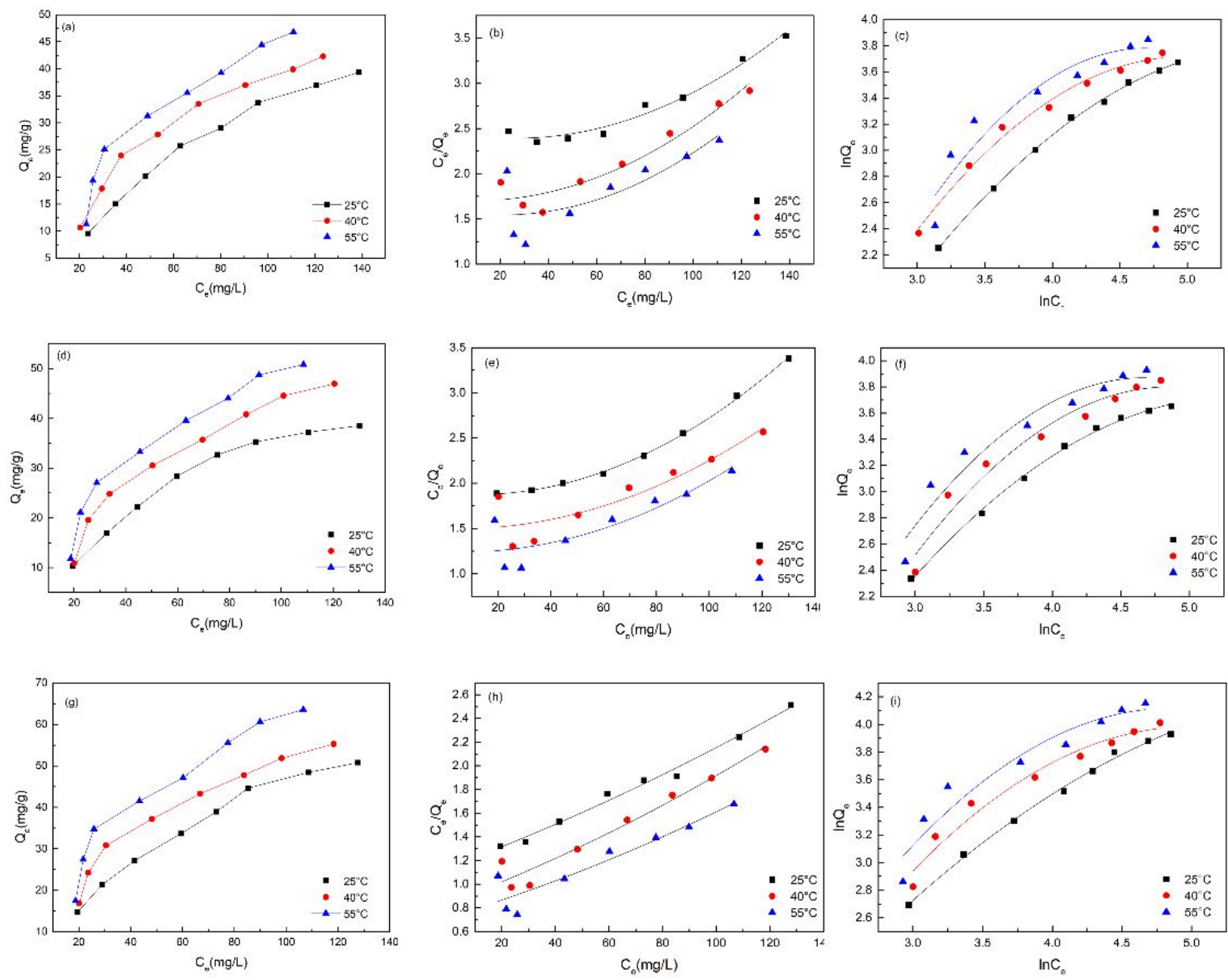

Figure S7 Adsorption isothermal curves of $\mathrm{Cr}(\mathrm{VI})$ on MWCNTs-3 (a), adsorption data of

MWCNTs-3 Langmuir model at different temperatures (b), adsorption data of MWCNTs-3

Freundlich model at different temperatures (c), adsorption isothermal curves of $\mathrm{Cr}(\mathrm{VI})$ on MWCNTs-OH-3 (d), adsorption data of MWCNTs-OH-3 Langmuir model at different temperatures (e), adsorption data of MWCNTs-OH-3 Freundlich model at different 
temperatures (f), adsorption isothermal curves of $\mathrm{Cr}(\mathrm{VI})$ on MWCNTs-COOH-3 (g), adsorption data of MWCNTs-COOH-3 Langmuir model at different temperatures (h), adsorption data of MWCNTs-COOH-3 Freundlich model at different temperatures (i)
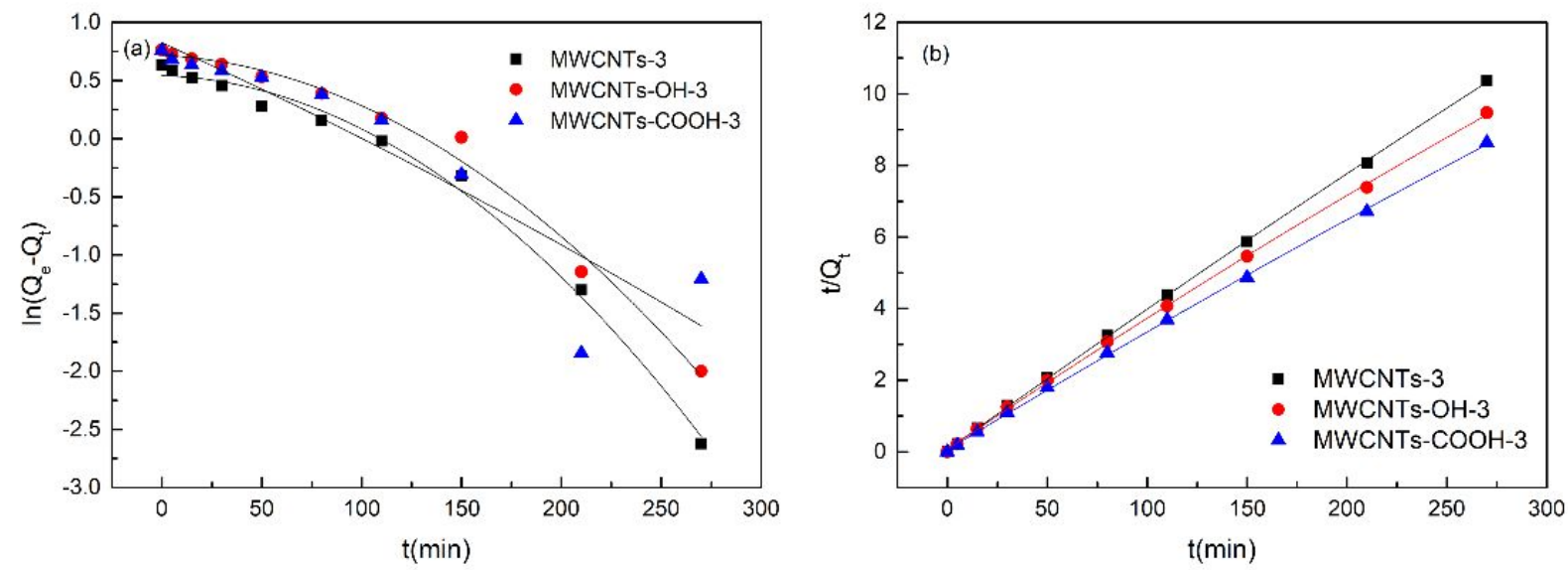

Figure S8 Pseudo first-order kinetic model for adsorption of $\mathrm{Cr}(\mathrm{VI})$ by MWCNTs immobilized ILs (a), Pseudo second-order kinetic model for adsorption of $\mathrm{Cr}(\mathrm{VI})$ by MWCNTs immobilized ILs (b) 